\title{
ELECTRIC-FIELD INDUCED INCREASE IN PARACELLULAR VASCULAR PERMEABILITY
}

\author{
Kaushik K. Rangharajan ${ }^{1}$, Prashanth Mohanasundaram ${ }^{l}$, Jennifer Morris, ${ }^{1}$ Ehsan Akbari ${ }^{l}$, \\ Griffin B. Spychalski ${ }^{l}$, Jonathan W. Song ${ }^{l, *}$, and Shaurya Prakash ${ }^{1, *}$ \\ ${ }^{1}$ The Ohio State University, Columbus, Ohio, USA
}

\begin{abstract}
We report a state-of-the-art microfluidic model of a branching blood vessel to study the role of simultaneous fluidic and electrical stimuli in regulating endothelial permeability. Previous efforts to modulate endothelial permeability were concentrated on the role of chemical factors and/or local fluidic forces as opposed to electric field inside in vitro systems. In this study, we report that addition of electric fields (e-field) over pre-existing flow (1 $\mu 1 / \mathrm{min})$ increases the endothelial permeability by $\sim 10$ times in comparison to no flow/no e-field control, accompanied by morphological changes to the endothelial cytoskeleton and ultrastructure.
\end{abstract}

\section{INTRODUCTION}

Tissue homeostasis is maintained by transport of oxygen, nutrients, and removal of waste across the body through a network of blood vessels. Endothelial cells (ECs) lining the inside of blood vessels in vascular networks form a semi-permeable barrier, selectively allowing bidirectional transport of small molecules, ions, and fluid transfer between blood and interstitial tissues to regulate angiogenesis, muscle tone, and host-defense reactions processes essential to outcomes in cancer, wound healing, and cardiovascular pathologies. Loss of endothelial barrier functions, resulting in elevated vessel permeability, is specifically expressed during chronic inflammation, cancer, wound healing, and atherosclerosis [1].

Increased endothelial permeability is also demonstrated in venules and is naturally accompanied by angiogenesis [2]. Angiogenesis is a physiological process characterized by growth of blood vessels from existing vasculature and is critical to wound healing, fetal development, and organ regeneration [3-5]. However, excessive vessel growth is indicative of inflammatory disorders (elevated vessel permeability state), cancer, and eye diseases [6].

From a biophysical standpoint, ECs are continuously exposed to shear stress in a direction tangential to the surface due to blood flow, with a physiological magnitude in the range of 0.7 130 dynes $/ \mathrm{cm}^{2}$ [5]. Presence of fluid shear is associated with reduced vessel permeability [7] resulting from actin cytoskeleton re-organization [8]. In contrast, transvascular transport i.e., the flow between two endothelial junction gaps or clefts, is concomitant of elevated vessel permeability [5]. Expansion of vascular networks by angiogenesis was previously shown to be regulated by local fluid mechanics in a shear dependent manner [9, 10]. Notably, microfluidic models that study angiogenesis do not consider branching morphology, and hence the biomechanical effects occurring at vessel bifurcations due to flow stagnation remains uninvestigated.

Similarly, the role of electric stimuli in regulating endothelial paracellular permeability and the resulting changes to cellular and junction morphology remains minimally investigated. Endogenous e-fields exist in vivo and arise as a consequence of (a) ion and molecule transfer, leading to charge separation from cell to cell via gap junctions [11] and (b) selective ion transport through ion pumps and channels driving charge segregation [12]. Such endogenous e-fields also exist around vasculature, with a measured zeta potential at the surface of the EC in the $0.1-0.4 \mathrm{~V}$ range for both the aorta and vena cava [13]. Regulation of endogenous efields play a vital role in embryonic growth, where major morphological development events are preceded by specific e-field patterns $[14,15]$. Similarly, disruption of epithelial barrier during wound initiation enhances trans-epithelial potential difference, initiating directional migration of cells to facilitate wound healing [16]. Exogenously applied DC e-fields $(75-100 \mathrm{mV} / \mathrm{mm})$ have shown that human umbilical vein endothelial cell (HUVECs) reorient, and stimulate VEGF production which are indicative of preangiogenic response [17].

The purpose of this paper is to report the morphological alterations to ECs accompanying changes to endothelial permeability when subjected to simultaneous mechanical and electrical stimuli of physiologically relevant magnitude.

\section{METHODS \\ Microfluidic Cell Culture}

SU-8 mold of a branching vessel geometry was embedded on a silicon wafer using optical lithography techniques. Microfluidic channels are $50 \mu \mathrm{m}$ deep and consist of an inlet vessel $1300 \mu \mathrm{m}$ wide that bifurcates from a bifurcation point (BP) into two distinct branching vessel (BVA), each with a width of $500 \mu \mathrm{m}$ (Fig. 1A). The two branching vessels are separated by an extra-cellular matrix (ECM), with targeted aperture windows that facilitate an interface between the ECs and ECM, allowing for transvascular transport from the apical (BP, BVA) side to the Basal (collagen) side [18].

Cured PDMS, present over the silicon mold, was peeled, diced into distinct devices, and bonded via plasma bonding to glass. The devices were incubated inside an oven overnight and UV sterilized prior to collagen injection. Type-1 rat tail collagen was prepared and injected into the central collagen channel (Fig. 1A), where the apertures restrict collagen outflow/spill due to capillary forces [18]. The devices were incubated inside an oven at $37^{\circ} \mathrm{C}$ overnight to complete collagen polymerization.

After polymerization of collagen, the devices were seeded (Fig. 1B) with HUVECs, incubated at $37^{\circ} \mathrm{C}$ for $24 \mathrm{~h}$ to facilitate homogenous endothelial monolayer development (Fig. 1C).

\section{Simultaneous Perfusion and Electric Field S timulation}

Potential difference was applied using gold $(\mathrm{Au})$ electrodes between the inlet ports, and the collagen (ground), outlet ports (ground) to induce a constant DC e-fields of magnitude $70 \mathrm{mV} / \mathrm{mm}$. $3 \mathrm{ml} \mathrm{BD}$-syringes (Fisher Scientific) were mounted on a Harvard apparatus syringe pump and connected to the outlet tubing to perfuse the device at a constant flow rate.

\section{Estimation of Endothelial Permeability}

After treating the endothelial monolayer with mechanical and/or electrical stimuli inside the microfluidic devices, changes to endothelial permeability were measured by monitoring extravasation rate of $10 \mu \mathrm{M}$ FITC (Fluorescein isothiocyanate) conjugated Dextran dye from BP and BVA into the ECM under an elevated pressure head, mirroring physiological hyper-tension effects. Using Matlab, a custom-written code was written to analyze the time-lapse images to extract paracellular permeability 

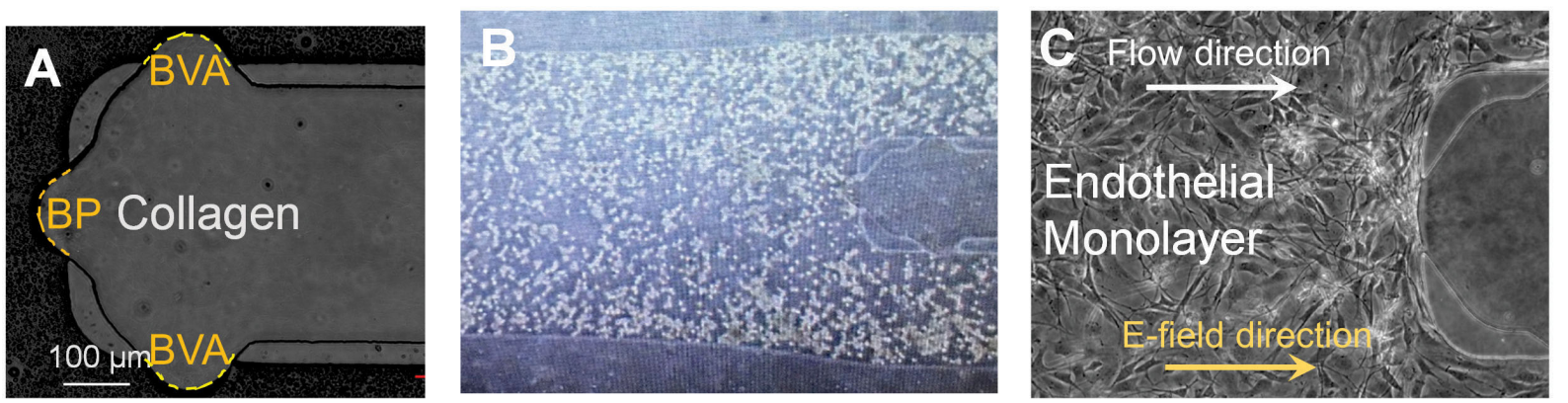

Figure 1: (A) Capillary driven collagen filling inside the microfluidic device, (B) Perfusion of endothelial cells inside the device, and $(C)$ Formation of endothelial monolayers after $24 \mathrm{~h}$ of incubation. White arrows indicate direction of fluid flow, and red arrows indicate direction of electric field.

[18]. For each case, overall transendothelial transport of $10 \mathrm{kDa}$ FITC-Dextran into the ECM over a period of 1 min was estimated by correlating the enhancement in intensity to enhancement in FITC-dextran concentration. Inside an Eulerian control volume, defined inside the collagen, the rate of enhancement in fluorescent signal was quantified for the tested cases to estimate the transendothelial flowrate and permeability.

\section{Immunofluorescence}

Immunofluorescence protocol to stain the endothelial F-actin with Alexa 488-Phalloidin and the nucleus with DAPI for confocal imaging follows from our previous reported protocol [18].

\section{S canning Electron Microscopy}

After experiments, devices were injected thrice with 1X PBS, followed by injection of fixing buffer $(2.5 \%$ glutaraldehyde in $0.1 \mathrm{M}$ phosphate buffer with $0.1 \mathrm{M}$ sucrose, $\mathrm{pH}$ 7.4). The cells were fixed for a duration of 30 mins, following which they were serially dehydrated using ethanol. Briefly, the devices were injected sequentially with $50 \%$ (1:1 ethanol: water), $70 \%, 80 \%$, $90 \%$ ethanol, with a total incubation time of 10 mins for each injection. Note: After the $90 \%$ ethanol injection, no water-based solution should be introduced into the device. Next, three $10 \mathrm{~min}$ injections with $100 \%$ ethanol were conducted.

After the third ethanol injection, a mixture of HMDS (Hexamethyldisilazane) and ethanol at a volumetric ratio of 1:3, 1:1, and 3:1 were prepared. First, the 1:3 HMDS:ethanol was injected into the device for 15 mins followed by a 15 min injection of the 1:1, and then the 3:1 HMDS:ethanol. Lastly, three 15 min injections with $100 \%$ HMDS were conducted, followed which the device was left to air-dry inside a biohood overnight. With a surgical blade, a small incision was made along the periphery of the microfluidic device at the PDMS-glass interface, following which the PDMS was peeled off from the glass, diced into thin sections, sputtered with $15 \mathrm{~nm}$ Nickel-Palladium, and mounted for SEM imaging.

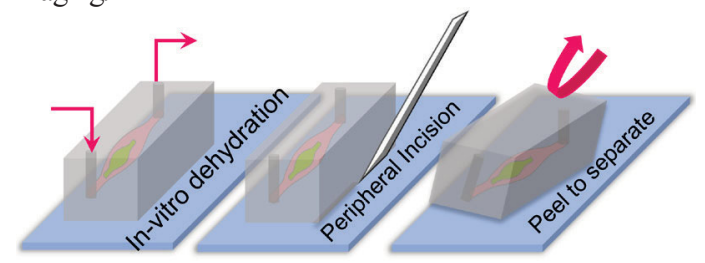

Figure 2: In vitro sample dehydration (increasing dosage from $50 \%$ to $100 \%$ ethanol, followed by HMDS:ethanol dehydration, $33 \%-100 \%$ by volume) and detachment methodology of bonded microfluidic device for direct observation of endothelial monolayer via SEM.

\section{RESULTS}

Shear $\mathrm{S}$ tress and $\mathrm{S}$ tagnation Pressure

For a flowrate of $1 \mu \mathrm{l} / \mathrm{min}$, the shear stress distribution in the branching vessel and at the stagnation point was estimated by multiplying the local shear rate with viscosity of media. The magnitude of shear stress was $0.3 \mathrm{dyn} / \mathrm{cm}^{2}$ at BVA apertures and negligible at BP (due to flow stagnation). Additionally, the magnitude of stagnation pressure was estimated to be $3.8 \mathrm{dyn} / \mathrm{cm}^{2}$ at the BP aperture.

\section{Extravasation of Dextran into ECM}

After application of simultaneous $1 \mu 1 / \mathrm{min}$ flow and $70 \mathrm{mV} / \mathrm{mm}$, the device permeability was estimated next by tracking the intravasation rate of Dextran dye into the collagen. For the same pressure gradient between BVA and collagen, enhanced extravasation of Dextran dye into the ECM compared to the control was observed (without flow or e-field, Fig. 3A), indicative of $\sim 10$ fold increase in permeability with application of $70 \mathrm{mV} / \mathrm{mm}$ over $1 \mathrm{~h}$ (Fig. 3B).
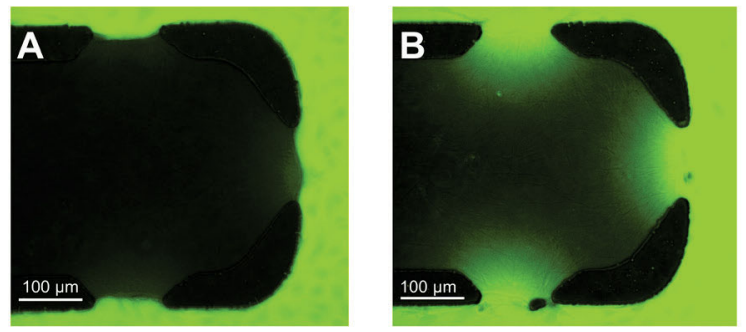

Figure 3: Intravasation of FITC-tagged Dextran from the Branching vessel and stagnation point in to the ECM over a duration of $1 \mathrm{~min}$. (A) Compared to the no fluid flow/no electric field control, (B) 10 fold enhancement in endothelial permeability was observed under the application of $1 \mathrm{~V}$ potentialdifference with a simultaneous flow of $1 \mu \mathrm{l} / \mathrm{min}$.

\section{Cytoskeleton-structure}

Changes to endothelial cytoskeleton structure were observed by imaging the F-actin alignment in the presence and absence of efield. Under the application of $70 \mathrm{mV} / \mathrm{mm}$ e-field over a duration of $1 \mathrm{~h}$, the orientation of F-actin filaments was found to align perpendicular to the direction of e-field [17] compared to randomly directed orientation of F-actin filaments in control.

\section{Effective cell-foot print}

SEM images revealed a $\sim 3 x$ decrease in effective cellfootprint area (Fig. 4A) with application of $70 \mathrm{mV} / \mathrm{mm}$, compared to the static no flow/no e-field control (Fig. 5A). 

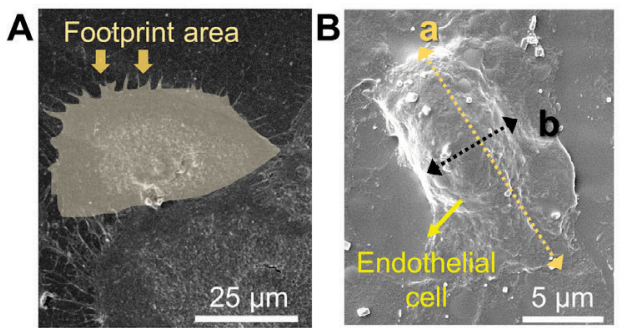

Figure 4: Representative SEM images of HUVECs of (A) control, (B) electric field.

\section{Shape Factor}

Quantification of the ratio of short to long axis $(b / a)$ of a cell (Fig. 4B) indicated effective circularity increased from 0.46 (control, elliptical) to 0.76 when an e-field of $70 \mathrm{mv} / \mathrm{mm}$ was applied (Fig. 5B).
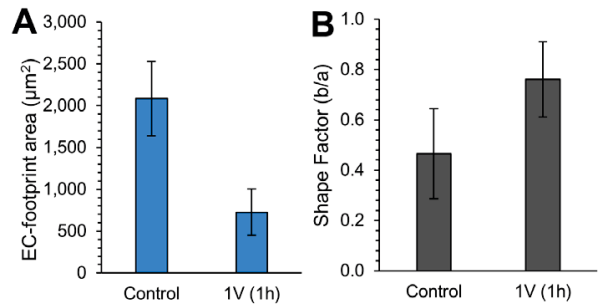

Figure 5: (A) Compared to control (no flow/no e-field), electric stimulation of $70 \mathrm{mV} / \mathrm{mm}$ for $1 \mathrm{~h}$ results in $3 x$ reduction in footprint area. EC footprint area was determined from multiple SEM images. (B) Shape factor increases from 0.46 for control (elliptical) to 0.76 (near-spherical) under an e-field of $70 \mathrm{mV} / \mathrm{mm}$ (Anova, $F=18.5, p<0.001$ ).

In summary, a novel microfluidic platform to regulate endothelial permeability under simultaneous mechanical and electrical stimuli is reported. Modifications to the endothelial ultrastructure facilitating the changes to permeability were additionally quantified.

\section{ACKNOWLEDGMENTS}

This work was supported by The American Heart Association (15SDG25480000), National Institute of Health (NHLBI R01HL141941), and the Center for Emergent Materials, an NSFMRSEC, grant DMR-1420451, the Center for Exploration of Novel Complex Materials, and the Institute for Materials Research. The authors acknowledge computational support and research license from the Ohio Supercomputer Center (OSC), the OSU Nanotech West cleanroom staff for assistance in fabrication, and the OSU Campus Microscopy and Imaging Facility (CMIF) for assistance with confocal microscopy.

\section{REFERENCES}

[1] J.A. Nagy, L. Benjamin, H. Zeng, A.M. Dvorak and H.F. Dvorak, "Vascular permeability, vascular hyperpermeability and angiogenesis", Angiogenesis, 11, 109 (2008).

[2] L. Claesson-Welsh, "Vascular permeability-the essentials", Upsala Journal of Medical Sciences, 120, 135 (2015).

[3] C. Patterson and M.S. Runge, "Therapeutic angiogenesis", Circulation, 99, 2614 (1999).

[4] E.W.K. Young and C.A. Simmons, "Macro- and microscale fluid flow systems for endothelial cell biology", Lab on a Chip, 10, 143 (2010).

[5] W.J. Polacheck, R. Li, S.G.M. Uzel, and R.D. Kamm,
"Microfluidic platforms for mechanobiology", Lab on a Chip, 13, 2252 (2013).

[6] J. Folkman "Angiogenesis: an organizing principle for drug discovery?”, Nature Reviews Drug Discovery, 6, 273 (2007).

[7] G.M. Price, K.H.K Wong, J.G. Truslow, A.D. Leung, C. Acharya and J. Tien, "Effect of mechanical factors on the function of engineered human blood microvessels in microfluidic collagen gels", Biomaterials, 31, 6182, (2010).

[8] E.A. Osborn, A. Rabodzey, C.F. Dewey and J.H. Hartwig, "Endothelial actin cytoskeleton remodeling during mechanostimulation with fluid shear stress", American Journal of Phy siology-Cell Phy siology, 290, 444 (2006).

[9] J.W. Song and L.L. Munn, "Fluid forces control endothelial sprouting", Proceedings of the National Academy of Sciences, 108, 15342 (2011).

[10] P.A. Galie, D-H.T. Nguyen, C.K. Choi, D.M. Cohen, P.A. Janmey and C.S. Chen, "Fluid shear stress threshold regulates angiogenic sprouting", Proceedings of the National Academy of Sciences, 111, 7968 (2014).

[11] R.H.W. Funk, "Endogenous electric fields as guiding cue for cell migration", Frontiers in Phy siology, 6, 143 (2015).

[12] H. Bai, J.V. Forrester and M. Zhao, "DC electric stimulation upregulates angiogenic factors in endothelial cells through activation of VEGF receptors", Cytokine,110, (2011).

[13] P.N. Sawyer, E. Himmelfar, I. Lustrin and H. Ziskind, "Measurement of streaming potentials of mammalian blood vessels, aorta and vena cava, in Vivo", Biophysical Journalm 6, 641, (1966).

[14] C.D. McCaig, A.M. Rajnicek, B. Song and M. Zhao, "Controlling cell behavior electrically: Current views and future potential", Physiological Reviews, 85,943, (2005).

[15] M. Levin, "Endogenous bioelectrical networks store nongenetic patterning information during development and regeneration", The Journal of Phy siology, 592, 2295 (2014).

[16] M. Zhao, "Electrical fields in wound healing - An overriding signal that directs cell migration", Seminars in Cell \& Developmental Biology, 20, 674, (2009).

[17] M. Zhao, H. Bai, E. Wang, J.V. Forrester, and C.D. McCaig, "Electrical stimulation directly induces pre-angiogenic responses in vascular endothelial cells by signaling through VEGF receptors." Journal of cell science, 117, 397, (2004).

[18] E. Akbari, G.B. Spychalski, K.K. Rangharajan, S. Prakash and J.W. Song, "Flow dynamics control endothelial permeability in a microfluidic vessel bifurcation model", Lab on a Chip, 18, 1084 (2018).

\section{CONTACT}

*J.W. Song; E-mail: song.1069@osu.edu

*S. Prakash; E-mail: prakash.31@osu.edu 